\title{
LETTERS
}

\section{Prevalence of anti-endothelial cell antibodies in idiopathic pulmonary arterial hypertension}

\section{To the Editors:}

Pulmonary arterial hypertension (PAH) is a rare disease often resulting in right-sided heart failure and premature death [1]. $\mathrm{PAH}$ is idiopathic (IPAH), heritable, or related to conditions such as connective tissue diseases (CTD) [2]. IPAH prognosis remains poor despite improved patient survival with current treatment options. Therefore, elucidating the pathophysiology of IPAH is important for the discovery of novel therapeutic approaches. Inflammation and immune reactivity have been implicated in the pathophysiology of IPAH. In 2005, TAMBY et al. [3] described the presence of anti-endothelial cell antibodies (AECA) in IPAH, pointing at the involvement of humoral immunity. AECA are a heterogeneous family of auto-antibodies capable of reacting with different endothelial cell (EC) structures [4]. In PAH, pulmonary EC dysfunction is considered a key player in the initiation and progression of the disease [5]. Systemic sclerosis (SSc) is a paradigm of autoimmune $\mathrm{PAH}$, as $10-15 \%$ of these patients develop PAH [6]. Interestingly, in SSc, immunoglobuliln (Ig)G AECA targeting cell-surface antigens have indeed been shown to induce EC dysfunction [7, 8]. Thus, IgG AECA-induced endothelial dysfunction may be the initiating event in IPAH. Whereas IgG auto-antibodies are considered pathogenic in various autoimmune diseases, $\operatorname{IgM}$ auto-antibodies have been proposed to be protective in these diseases [9].

Regarding a pathophysiological role of AECA, it is important to identify whether AECA are reactive with EC-surface antigens. To corroborate and further investigate the role of the humoral immune system in IPAH, we aimed to study the prevalence of IgG, as well as IgM AECA targeting cell-surface EC antigens using a cell-based ELISA with viable human umbilical vein EC (HUVEC).

We screened five study cohorts for the presence of AECA. 1) 29 IPAH patients; mean pulmonary artery pressure $\left(\bar{P}_{\text {pa }}\right)$ $45.5 \mathrm{mmHg}$ as measured by right heart catheterisation. 2) 18 CTD-associated PAH patients (14 SSc, two systemic lupus erythematosus and two mixed connective tissue disease); $\bar{P}_{\text {pa }}$ $42.5 \mathrm{mmHg}$ as measured by right heart catheterisation; total lung capacity (TLC) $>70 \%$ of predicted. 3) 51 SSc patients without PAH (nine diffuse SSc and 42 limited SSc); right systolic ventricular pressure $<35 \mathrm{mmHg}$, as estimated by echocardiography; TLC $>70 \%$ pred. 4) 40 type II diabetes mellitus (DM II) patients with vascular involvement. 5) 40 healthy individuals. The CTD-associated PAH patients and SSc patients without $\mathrm{PAH}$ were included as a comparison cohort with known immunopathological involvement. DM II patients served as controls with known vascular dysfunction. The healthy individuals were included to determine optimal cut-off values for
AECA positivity. All subjects gave their informed consent prior to participation. Age was similar in all five study cohorts $(p=0.18)$. Previous use of immunosuppressive drugs occurred in four out of $18 \mathrm{CTD}$-associated PAH patients as compared with 14 out of 51 in SSc and zero out of 29 in IPAH patients.

HUVEC isolation and culture was carried out as previously described [10]. Experiments were executed with confluent HUVEC monolayers between passages 3 and 5. HUVEC monolayers displayed the typical cobblestone morphology of ECs. HUVEC were phenotypically characterised as ECs by flow cytometry: CD31+ (96.5\%), CD146+ (98.6\%), Ulex europaeus lectin positive $(99.3 \%)$, von Willebrand factor positive (98.9\%) and CD90- (0.3\%).

A cyto-ELISA with unfixed HUVEC was performed to detect AECA specifically targeting EC-surface antigens [10]. Experiments were performed three times in triplicate. Cut-off points based on the optical density (OD) values from the IPAH cohort compared to the healthy individuals were calculated using receiver operating characteristic curve analysis. Sera were considered positive for IgG and IgM AECA if the OD value was $>0.322$ and 0.137 , respectively. The intra- and interassay coefficients of variation for the HUVEC ELISA were $5.15 \%$ and $19.89 \%$, respectively. The mean kappa concordance value of the HUVEC ELISA was 0.87 ( $\mathrm{p}<0.0001)$.

IgG AECA prevalence in the IPAH cohort was $62.1 \%$ (18 out of 29 ) compared to $77.8 \%$ (14 out of 18 ) in the CTD-associated $\mathrm{PAH}$ and $43.1 \%$ (23 out of 51 ) in the SSc without PAH cohorts (table 1). IgG AECA prevalence in the IPAH cohort did not differ significantly from that of the CTD-associated PAH and SSc without PAH cohorts ( $p=0.261$ and $p=0.104$, respectively). Of the 18 patients with CTD-associated PAH, 14 were diagnosed with SSc. The prevalence of IgG AECA in these 14 SSc associated PAH patients was $71.4 \%$ (10 out of 14 ). IgG AECA prevalence in the IPAH and CTD-associated PAH cohorts was significantly higher compared to the DM II cohort $(\mathrm{p}<0.0001$ and $\mathrm{p}<0.0001$, respectively) and the healthy individuals $(\mathrm{p}=0.015$ and $\mathrm{p}=0.001$, respectively).

AECA levels of the SSc-associated PAH cohort were significantly higher in comparison to SSc patients without PAH $(p=0.011)$. The use of immunosuppressive drugs had no significant impact on IgG AECA levels.

IgM AECA prevalence in the IPAH cohort was 44.8\% (13 out of 29 ) compared to $61.1 \%$ (11 out of 18 ) in the CTD-associated PAH cohort and $41.2 \%$ (21 out of 51) in the SSc without PAH cohort (table 1). IgM AECA prevalence in the IPAH cohort did not differ significantly from that of the CTD-associated PAH and SSc without PAH cohorts $(p=0.278$ and $p=0.751$, 


\begin{tabular}{|c|c|c|c|}
\hline \multirow[t]{3}{*}{ TABLE 1} & \multirow{3}{*}{ Patients n } & $\begin{array}{l}\text { of patien } \\
\text { ulin (lg)G } \\
\text { s (AECA) } \\
\text { on hum } \\
\text { JEC) }\end{array}$ & $\begin{array}{l}\text { id healthy } \\
\text { lgM anti- } \\
\text { cted } \\
\text { nbilical }\end{array}$ \\
\hline & & \multicolumn{2}{|c|}{ Immunoglobulin type } \\
\hline & & $\lg G$ & $\lg M$ \\
\hline IPAH & 29 (17 females) & $18(62.1)$ & $13(44.8)$ \\
\hline CTD-associated PAH & 18 (17 females) & $14(77.8)$ & $11(61.1)$ \\
\hline SSc & 14 & 10 & 9 \\
\hline SLE & 2 & 2 & 1 \\
\hline MCTD & 2 & 2 & 1 \\
\hline SSc & 51 (41 females) & $22(43.1)$ & 21 (41.2) \\
\hline ISSc & 42 & 20 & 17 \\
\hline dSSc & 9 & 2 & 4 \\
\hline DM II & 40 (28 females) & $5(12.5)$ & $7(17.5)$ \\
\hline $\mathrm{HC}$ & 40 (22 females) & $13(32.5)$ & $8(20.0)$ \\
\hline \multicolumn{4}{|c|}{$\begin{array}{l}\text { Data are presented as } n \text { or } n(\%) \text {, unless otherwise stated. IPAH: idiopathic } \\
\text { pulmonary arterial hypertension; CTD-associated PAH: connective tissue } \\
\text { disease-associated pulmonary arterial hypertension; SSc: systemic sclerosis; } \\
\text { SLE: systemic lupus erythematosus; MCTD: mixed connective tissue disease; } \\
\text { ISSc: limited cutaneous SSc; dSSc: diffuse cutaneous SSCs; DM II: diabetes } \\
\text { mellitus type II; HC: healthy controls. }\end{array}$} \\
\hline
\end{tabular}

respectively). IgM AECA prevalence in the IPAH and CTDassociated PAH cohorts was significantly higher in comparison to the DM II cohort ( $p=0.014$ and $p=0.001$, respectively) and the healthy individuals ( $p=0.027$ and $p=0.002$, respectively).

Again, the use of immunosuppressive drugs had no significant impact on IgM AECA levels.

In the IPAH cohort the $\operatorname{IgG/IgM}$ ratio correlated with pulmonary vascular resistance $(\mathrm{R}=0.433, \mathrm{p}=0.039)$ and correlated inversely with cardiac index $(R=-0.420, p=0.046)$. Such a correlation could not be demonstrated in the CTD-associated PAH cohort.

AECA are present in patients with established PAH. Our data corroborate the findings described by TAMBY et al. [3]. Moreover, by using a cell-based ELISA with viable ECs, we detected functionally relevant AECA able to target cell-surface antigens. Dysfunction of pulmonary ECs is considered pivotal in IPAH pathogenesis. The initiating stimulus or injury resulting in EC dysfunction, however, is still a matter of speculation. We hypothesise that an autoimmune attack by IgG AECA may be the initial trigger leading to EC dysfunction. However, IgG AECA was not found in all PAH patients. There may be several explanations for this. First, the phenotype expressed by the EC is of crucial importance. Ideally, patients' own pulmonary ECs should be used to detect AECA. These ECs may harbour the unique endothelial epitope phenotype influenced by individual factors, such as shear stress, viral infections and pulmonary inflammatory characteristics (e.g. cytokine profile). To better mimic the pulmonary environment we also determined the prevalence of IgG AECA on normal human pulmonary artery ECs. No difference in AECA prevalence was found compared to the results obtained by the HUVEC ELISA (data not shown). Secondly, some PAH patients were treated with immunosuppressants that may have suppressed AECA levels. However, no statistically significant difference was observed between AECA levels of treated and untreated patients.

It may be questioned whether AECA in IPAH play a primary pathogenic role or whether they arise as a consequence of EC damage. The observation of low prevalence of IgG AECA in our diabetic control group with established vascular damage supports a primary role for IgG AECA in IPAH. The higher IgG AECA prevalence in SSc patients with $\mathrm{PAH}$ compared to SSc patients without PAH also supports their pathogenic role. The role of IgM AECA is also unclear. IgM AECA prevalence in IPAH, CTD-associated PAH, and SSc without PAH did not differ. However, the finding that the $\operatorname{IgG} / \operatorname{IgM}$ AECA ratio correlates with severity of disease in the IPAH cohort suggests that IgM AECA may be protective, for instance by binding to relevant endothelial epitopes, thus hindering IgG binding.

The mechanisms by which IgG AECA might lead to EC dysfunction are also incompletely understood. In SSc, which is considered a paradigm of autoimmune $\mathrm{PAH}$, AECA targeting EC-surface antigens have been shown to upregulate adhesion molecule expression and to induce EC apoptosis [7, 8]. Moreover, it is possible that downstream signalling may have an impact on adjacent pulmonary fibroblasts. However, our study does not address the functional aspects of IgG AECA. Therefore, further studies to define putative pathogenic potentials of IgG AECA in IPAH are required.

In conclusion, we report the presence of $\operatorname{IgM}$ and IgG AECA specifically targeting EC-surface antigens in IPAH, further corroborating the involvement of autoimmune processes in the pathophysiology of this disease.

Special thanks go to H. Bilo (Dept of Internal Medicine/Diabetes Centre, Isala Clinic, Zwolle, the Netherlands) who provided the serum samples from the diabetes mellitus type II patients.

\section{S.J. Arends*, J. Damoiseaux*, A. Duijvestijn*,} L. Debrus-Palmans*, K. Boomars", B. Broers ", J.W. Cohen Tervaert* and P. van Paassen*

*Dept of Internal Medicine/Division of Clinical \& Experimental Immunology, CARIM, "Dept of Respiratory Diseases, and "Dept of Cardiology, CARIM, Maastricht University Medical Centre, Maastricht, The Netherlands.

Correspondence: S.J. Arends, Dept of Internal Medicine/ Division of Clinical \& Experimental Immunology, Maastricht University Medical Centre, P.O. Box 616, 6200 MD Maastricht, The Netherlands. E-mail: steven.arends@immuno.unimaas.nl

Statement of Interest: Statements of interest for J. Damoiseaux and for the study itself can be found at www.erj.ersjournals. $\mathrm{com} / \mathrm{misc} /$ statements.dtl

\section{REFERENCES}

1 Rubin L. Primary pulmonary hypertension. N Engl J Med 1997; 336: 111-117. 
2 Simonneau G, Robbins I, Beghetti M, et al. Updated clinical classification of pulmonary hypertension. J Am Coll Cardiol 2009; 54: Suppl. S, S43-S54.

3 Tamby M, Chanseaud Y, Humbert M, et al. Anti-endothelial cell antibodies in idiopathic and systemic sclerosis associated pulmonary arterial hypertension. Thorax 2005; 60: 765-772.

4 Belizna C, Duijvestijn A, Hamidou M, et al. Antiendothelial cell antibodies in vasculitis and connective tissue disease. Ann Rheum Dis 2006; 65: 1545-1550.

5 Humbert M, Montani D, Perros F, et al. Endothelial cell dysfunction and cross talk between endothelium and smooth muscle cells in pulmonary arterial hypertension. Vasc Pharmacol 2008; 49: 113-118.

6 Hesselstrand R, Ekman R, Eskilsson J. Screening for pulmonary hypertension in systemic sclerosis: the longitudinal development of tricuspid gradient in 227 consecutive patients, 1992-2001. Rheumatology 2005; 44: 366.
7 Bordron A, Dueymes M, Levy Y, et al. The binding of some human antiendothelial cell antibodies induces endothelial cell apoptosis. J Clin Invest 1998; 101: 2029-2035.

8 Mihai C, Cohen Tervaert JW. Anti-endothelial cell antibodies in systemic sclerosis. Ann Rheum Dis 2010; 69: 319-324.

9 Witte T, Hartung K, Sachse C, et al. IgM anti-dsDNA antibodies in systemic lupus erythematosus: negative association with nephritis. Rheumatol Int 1998; 18: 85-91.

10 van Paassen P, Duijvestijn A, Debrus-Palmans L, et al. Induction of endothelial cell apoptosis by IgG antibodies from SLE patients with nephropathy: A potential role for anti-endothelial cell antibodies. Ann NY Acad Sci 2007; 1108: 147-156.

\section{Levels of soluble human leukocyte antigen-G are increased in asthmatic airways}

\section{To the Editors:}

Human leukocyte antigen-G (HLA-G) is a non-classical, class $\mathrm{Ib}$, major histocompatibility complex antigen, encoded by a gene on chromosome 6p21 within the HLA complex [1]. HLA-G is constitutively expressed during pregnancy where it has a critical role in maintaining immune tolerance toward the allogenic fetus and placenta $[2,3]$, but has also been associated with inflammatory diseases such as psoriasis, multiple sclerosis, and ulcerative colitis, and with solid-organ transplantation [3, 4]. We recently reported associations between variation in HLA-G and risk for asthma in Chicago-area asthma families, in multigenerational Dutch asthma families and in a birth cohort at high risk for developing asthma [1, 5]. A role for HLA-G in asthma pathogenesis was further suggested by the demonstration of expression of a soluble isoform of HLA-G, sHLA-G5, in airway epithelial cells [1] and of increased circulating plasma levels of sHLA-G in children with atopic asthma [6]. Because airway inflammation in asthma involves a T-helper cell (Th) type 2-skewing of lymphocytes similar to pregnancy, HLA-G is an attractive candidate molecule for promoting the immune profile characteristic of asthma. Localisation of HLA-G to airway epithelium suggests that its dysregulation could contribute to airway inflammation in chronic asthma. To evaluate this further, we hypothesised that HLA-G abundance would be increased in asthmatic airways.

To test this hypothesis, we measured concentrations of sHLA-G in bronchoalveolar lavage (BAL) fluid obtained from 12 non-asthmatic control subjects and 15 subjects with mild persistent asthma. The use of human subjects was approved by the University of Chicago Institutional Review Board (Chicago, IL, USA). Asthma was diagnosed using National Asthma Education and Prevention Program guidelines. Subjects with a smoking history of $\geqslant 10$ pack $\cdot \mathrm{yrs}^{-1}$, who had used oral corticosteroids within 6 months of study, who had received emergent care or had been hospitalised for asthma within 6 months of study, were excluded. Bronchoscopy was done at a time of stability for each subject.

The demographic, clinical and pulmonary function data for the subjects in our study are presented in table 1. As expected, subjects with asthma had a lower forced expiratory volume in $1 \mathrm{~s} \%$ predicted $(p=0.01)$, more atopy $(p=0.0001)$ and more peripheral blood eosinophils $(p=0.02)$ compared with control subjects. However, there were no significant differences in cells counts in bronchoalveolar lavage (BAL) fluid between the two groups. Lavage fluid was concentrated approximately 30-fold using Centriprep ultra-filtration chambers (Millipore, Inc., Billerica, MA, USA) with a 3-kD molecular weight cut-off filter. The retentate was analysed for the presence of sHLA-G using an ELISA assay (Exbio, Inc., Vestec, Czech Republic). The capture antibody, MEM-G/9, recognises shed G1 and secreted G5, and the secondary antibody, anti- $\beta 2 \mathrm{~m}$, ensures measurement of $\beta 2 \mathrm{~m}$-configured soluble G [7]. The limit of sensitivity was $\sim 0.2 \mathrm{U} \cdot \mathrm{mL}^{-1}$. Values were adjusted for the degree of concentration as noted above and expressed as $\mathrm{U} \cdot \mathrm{mL}^{-1} \mathrm{BAL}$ fluid.

sHLA-G levels were increased in the BAL fluid of 15 asthmatic subjects (median 6.8 (interquartile range, 2.8-7.8) $\mathrm{U} \cdot \mathrm{mL}^{-1}$ ) compared with 12 control subjects (median 1.6 (1.0 3.0) $\mathrm{U} \cdot \mathrm{mL}^{-1}, \mathrm{p}=0.01$ by Mann-Whitney test) (fig. 1). One control value and no asthmatic values were below the limit of detection for the assay. We also examined whether racial background accounted for the observed differences in sHLA-G. There was no significant difference in sHLA-G levels in nine Caucasian asthmatic subjects (median 5.6 (1.56.9) $\mathrm{U} \cdot \mathrm{mL}^{-1}$ ) versus six African-American asthmatic subjects (median $6.9(6.2,8.6) \mathrm{U} \cdot \mathrm{mL}^{-1}, \mathrm{p}=0.24$ by Mann-Whitney test). There were too few African-American control subjects for analysis in that group. 